\title{
On Fréchet differentiability of multifunctions ${ }^{\dagger}$
}

\author{
VALENTIN V. GOROKHOVIK* and PETER P. ZABREIKO \\ Institute of Mathematics, The National Academy of Sciences of Belarus, \\ Surganova st., 11, Minsk 220072, Belarus
}

(Received 20 February 2004; in final form 14 December 2004)

\begin{abstract}
The purpose of our article is to extend the classical notion of Fréchet differentiability to multifunctions. To this end we define the notion of affinity for multifunctions and study the basic properties of affine multifunctions. Then using affine multifunctions as local approximations and the Hausdorff distance for defining an approximation mode, we introduce the notion of Fréchet differentiability for multifunctions mapping points of a finite-dimensional normed space to compact convex subsets of another finite-dimensional normed space. We characterize Fréchet differentiability of multifunctions through the differentiable properties of their support functions and discuss the relationship of our notion of differentiability with other ones which were studied by Blagodatskikh (Blagodatskikh, V.I., 1984, Maximum principle for differential inclusions. Trudy Matematicheskogo Instituta AN SSSR, 166, 23-43 (in Russian)), Rubinov (Rubinov, A.M., 1985, The conjugate derivative of a multivalued mapping and differentiability of the maximum function under connected constraints. Sibirskii Matematicheskii Zhurnal, 26(3), 147-155 (in Russian)), Tyurin (Tyurin, Yu. N., 1965, A mathematical formulation of a simplified model of industrial planning. Ekonomika i Matematicheskie Metody, 1(3), 391-409 (in Russian)), Banks and Jacobs (Banks, H.T. and Jacobs, M.Q., 1970, On differential calculus of multifunctions. Journal of Mathematical Analysis and Applications, 29(3), 246-272).
\end{abstract}

Keywords: Multifunction; Fréchet differentiability; Hausdorff metric; Affine multifunction; Difference-sublinear mappings

Mathematics Subject Classifications 2000: 49J53; 49J50; 58C06; 58C20

\section{Introduction}

At the present time, three approaches are mainly used to introduce the concepts of differentiability of multifunctions (multi-valued mappings, set-valued mappings, correspondences). The first approach is essentially a geometrical one. Its main idea may be presented as follows. A multi-valued mapping $F$ is identified with its graph gr $F$ and a derivative of $F$ at a point $z^{0} \in \operatorname{gr} F$ is defined as a multi-valued mapping

*Corresponding author. Email: gorokh@im.bas-net.by

$\uparrow$ The article is dedicated to Professor V.F. Demyanov on his 65 th birthday. 
whose graph is a tangent or normal (in one sense or another) cone to gr $F$ at $z^{0}$. We refer to [5-16] and others for the detailed presentation of different realizations of this approach.

The second approach that was developed in [17-22] and some other papers extends the classical scheme of definition of differentiability to multifunctions. In accordance with this scheme, one needs first to define a relation of tangency on the class of mappings under consideration and to choose a subclass of local (differential) approximations consisting of mappings which are 'simple' in some sense. Then a mapping $F$ is called differentiable if there exists a 'simple' mapping belonging to the chosen subclass of local approximations which is tangent to $F$. In the classical definitions of Gâteaux, Hadamard and Fréchet differentiability of single-valued mappings affine functions are used as local approximations and the linear mapping corresponding to the affine local approximation of a differentiable mapping is called a derivative.

The third approach is based on embedding multifunctions under consideration into a space of single-valued mappings, whose values belong to some functional space, which is endowed with a structure of topological vector space or even a structure of normed space. Whenever such embedding may be made, one may interpret multifunctions as single-valued mappings and use standard classicial definitions of differentiability. This approach was realized in [1-4,23-27] for multifunctions with convex and compact values. Such multifunctions are associated with their support functions and, in such a way, they are embedded into the space of single-valued mappings with values in the space of positively homogeneous and continuous functions which can be endowed with a structure of a Banach space (at least in finitedimensional setting). The main drawback of this approach is that the derivative and the corresponding local (differential) approximation are defined as single-valued mappings with values in the functional space and may have, in general, no inverse images among multifunctions.

The main purpose of the article is to extend the classical notion of Fréchet differentiability to multifunctions mapping points of a finite-dimensional normed space to compact convex subsets of another finite-dimensional normed space. To do it we follow the second approach described above. In section 3 we define a notion of affinity for multifunctions and study basic properties of affine multifunctions. Then in section 4, using affine multifunctions as local approximations and the Hausdorff distance for defining a tangency relation, we introduce the notion of Fréchet differentiability for multifunctions and present some properties of Fréchet differentiable multifunctions. In section 5, we characterize Fréchet differentiability of multifunctions through the differential properties of their support functions and discuss the relationship of our notion of differentiability with other ones which were studied by Blagodatskikh [1,28], Rubinov [2], Tyurin [3], Banks and Jacobs [4].

\section{Preliminaries}

Let $X$ and $Y$ be finite-dimensional normed vector spaces over reals $\mathbb{R}$.

A multifunction $F: X \rightrightarrows Y$ from $X$ into $Y$ assigns to each $x \in X$ a (possibly empty) subset $F(x) \subset Y$. The sets

$$
\operatorname{dom} F:=\{x \in X \mid F(x) \neq \emptyset\}
$$


and

$$
\operatorname{gr} F:=\{(x, y) \in X \times Y \mid y \in F(x)\}
$$

are called the effective domain and the graph of the multifunction $F$, respectively.

A multifunction $F: X \rightrightarrows Y$ is said to be closed if its graph gr $F$ is a closed subset of $X \times Y$.

A multifunction $G: X \rightrightarrows Y$ is called an extension (a restriction) of a multifunction $F: X \rightrightarrows Y$ if $\operatorname{dom} F \subset \operatorname{dom} G(\operatorname{dom} F \supset \operatorname{dom} G)$ and $F(x)=G(x)$ for all $x \in \operatorname{dom} F$ $(x \in \operatorname{dom} G)$.

By bcc $Y$ we denote the collection of bounded closed convex subsets of $Y$. The collection bcc $Y$ equipped with the addition (by Minkowski)

$$
M+N:=\left\{y_{1}+y_{2} \mid y_{1} \in M, y_{2} \in N\right\} \quad(M, N \in \operatorname{bcc} Y)
$$

and multiplication by nonnegative reals

$$
\alpha M:=\{\alpha y \mid y \in M\} \quad(M \in \operatorname{bcc} Y, \alpha \geq 0)
$$

is a semilinear space [29].

The function

$$
d_{H}(M, N):=\inf \left\{\alpha \geq 0 \mid M \subset N+\alpha B_{Y}, N \subset M+\alpha B_{Y}\right\}
$$

( $B_{Y}$ is the unit ball in $Y$ ) called the Hausdorff distance determines a structure of a metric space on bec $Y$.

Throughout this article we confine ourselves to multifunctions $F: X \rightrightarrows Y$ with $F(x) \in$ bec $Y$ for all $x \in \operatorname{dom} F$. It enables us to interpret the multifunction $F: X \rightrightarrows Y$ considered below as the single-valued function $F: X \rightarrow$ bcc $Y$ from the normed vector space $X$ into the semilinear metric space bcc $Y$.

Let $X^{*}$ and $Y^{*}$ be the norm duals of $X$ and $Y$ respectively.

By $H\left(Y^{*}\right)$ we denote the Banach space of positive homogeneous $\left(p\left(\lambda y^{*}\right)=\lambda p\left(y^{*}\right)\right.$ for all $y^{*} \in Y^{*}$ and all $\left.\lambda \geq 0\right)$ and continuous functions $p: Y^{*} \rightarrow \mathbb{R}$ with standard algebraic operations and the norm defined by

$$
\|p\|=\max _{\left\|y^{*}\right\|=1}\left|p\left(y^{*}\right)\right| .
$$

A positive homogeneous function $p: Y^{*} \rightarrow \mathbb{R}$ is said to be sublinear [30] if it is subadditive, i.e.,

$$
p\left(y_{1}^{*}+y_{2}^{*}\right) \leq p\left(y_{1}^{*}\right)+p\left(y_{2}^{*}\right) \quad\left(y_{1}^{*}, y_{2}^{*} \in Y^{*}\right)
$$

The collection $\mathrm{CH}\left(Y^{*}\right)$ of sublinear functions is a convex cone in $H\left(Y^{*}\right)$. It follows from [30, Theorem 3.1.5] that the cone $C H\left(Y^{*}\right)$ is closed in the norm topology of the Banach space $H\left(Y^{*}\right)$. The linear hull $D C H\left(Y^{*}\right)$ of the cone $C H\left(Y^{*}\right)$ is called 
the space of difference-sublinear functions; a function $p: Y^{*} \rightarrow \mathbb{R}$ lies in $D C H\left(Y^{*}\right)$ if and only if it can be presented as a difference of two sublinear functions.

A single-valued mapping $P: Y^{*} \rightarrow X^{*}$ is said to be dffierence-sublinear if for any $x \in X$ the real-valued function $y^{*} \rightarrow\left\langle x, P\left(y^{*}\right)\right\rangle$ is difference-sublinear. A detail account of results concerning difference-sublinear functions and mappings can be found in $[31-34]$.

The correspondence assigning to each subset $M \in$ bcc $Y$ its support function

$$
s_{M}\left(y^{*}\right)=\max \left\{\left\langle y, y^{*}\right\rangle \mid y \in M\right\}
$$

is an isometrical isomorphism (called the Minkowski duality [29]) between the metric sublinear space bcc $Y$ and the convex cone $C H\left(Y^{*}\right)$ of sublinear functions.

Given a multifunction $F: X \rightarrow$ bcc $Y$, a real-valued function $s_{F}: \operatorname{dom} F \times Y^{*} \rightarrow \mathbb{R}$ defined by

$$
s_{F}\left(x, y^{*}\right):=\max \left\{\left\langle y, y^{*}\right\rangle \mid y \in F(x)\right\}
$$

is called the support function of $F$.

Due to the Minkowski duality, one can associate with a multifunction $F: X \rightarrow$ bec $Y$ the single-valued mapping $\tilde{F}: \operatorname{dom} F \rightarrow C H\left(Y^{*}\right)$ such that $\tilde{F}(x)=s_{F}(x, \cdot)$ for all $x \in \operatorname{dom} F$. Since $C H\left(Y^{*}\right) \subset D C H\left(Y^{*}\right) \subset H\left(Y^{*}\right)$ one can also consider $\tilde{F}$ either as a mapping from dom $F$ into the normed space $D C H\left(Y^{*}\right)$ or as a mapping from $\operatorname{dom} F$ into the Banach space $H\left(Y^{*}\right)$.

\section{Affine multifunctions}

\subsection{Definition and elementary properties}

Let $X$ and $Y$ be finite-dimensional vector spaces over reals $\mathbb{R}$.

A multifunction $\mathcal{A}: X \rightarrow$ bcc $Y$ is said to be

(i) convex if

$$
\alpha \mathcal{A}\left(x_{1}\right)+(1-\alpha) \mathcal{A}\left(x_{2}\right) \subset \mathcal{A}\left(\alpha x_{1}+(1-\alpha) x_{2}\right)
$$

for all $x_{1}, x_{2} \in \operatorname{dom} \mathcal{A}$ and $\alpha \in[0,1]$;

(ii) affine if

$$
\alpha \mathcal{A}\left(x_{1}\right)+(1-\alpha) \mathcal{A}\left(x_{2}\right)=\mathcal{A}\left(\alpha x_{1}+(1-\alpha) x_{2}\right)
$$

for all $x_{1}, x_{2} \in \operatorname{dom} \mathcal{A}$ and $\alpha \in[0,1]$.

Convex multifunctions have been studied by many authors from different points of view (see, for instance, $[5,35,36]$ and references therein). Affine multifunctions were first introduced by Gautier [37] (see, also, Lemarechal and Zowe [20]) for the case $X=\mathbb{R}$ and $\operatorname{dom} \mathcal{A}=[0, T] \subset \mathbb{R}, T>0$. The definition of affinity presented here was given in [38]. 
Notice that for single-valued functions the inclusion (1) and the equality (2) are equivalent to each other. Moreover, the following statement is true.

Proposition 3.1 Let $\mathcal{A}: X \rightarrow$ bcc $Y$ be a convex multifunction. If $\mathcal{A}\left(x^{0}\right)$ is a singleton (i.e., $\left.\mathcal{A}\left(x^{0}\right)=\left\{y^{0}\right\}\right)$ for some point $x^{0} \in \operatorname{ri}(\operatorname{dom} \mathcal{A})$ then $\mathcal{A}: X \rightarrow \operatorname{bcc} Y$ is actually a single-valued affine function on $\operatorname{dom} \mathcal{A}$.

Here $\operatorname{ri}(\operatorname{dom} \mathcal{A})$ stands for the relative interior of $\operatorname{dom} \mathcal{A}$.

Proof Let $x$ be a point of $\operatorname{dom} \mathcal{A}$. Since $x^{0} \in \operatorname{ri}(\operatorname{dom} \mathcal{A})$ then for some $\bar{x} \in \operatorname{dom} \mathcal{A}$ and $\alpha \in(0,1)$ we have $x^{0}=\alpha x+(1-\alpha) \bar{x}$. From (1) it follows that $\alpha \mathcal{A}(x)+$ $(1-\alpha) \mathcal{A}(\bar{x}) \subset \mathcal{A}\left(x^{0}\right)=\left\{y^{0}\right\}$. This shows that both sets $\mathcal{A}(x)$ and $\mathcal{A}(\bar{x})$ are singletons. Since $x$ is an arbitrary point of $\operatorname{dom} \mathcal{A}$ then $\mathcal{A}(x)$ is single-valued for each $x \in \operatorname{dom} \mathcal{A}$ and, hence, by the above remark it follows that $\mathcal{A}$ is affine.

In general, however, affine multifunctions form a proper subclass of the class of convex multifunctions. Let us consider the multifunction $\mathcal{A}: \mathbb{R} \rightarrow$ bcc $\mathbb{R}$ with gr $\mathcal{A}:=\{(x, y) \in \mathbb{R} \times \mathbb{R} \| x|\leq y \leq-| x \mid+2\}$. It is not difficult to see that this multifunction is convex, but not affine.

Let $s_{\mathcal{A}}(\cdot, \cdot): X \times Y^{*} \rightarrow \mathbb{R}$ be the support function of a multifunction $\mathcal{A}: X \rightarrow$ bec $Y$. Straightforward from the above definitions and from properties of convex sets and their support functions (see, for instance, [30]), we obtain the following characterizations for both convex and affine multifunctions.

Proposition 3.2 [35-37] A multifunction $\mathcal{A}: X \rightarrow$ bec $Y$ is convex (affine) if and only if for each $y^{*} \in Y^{*}$ the real-valued function $s_{\mathcal{A}}\left(\cdot, y^{*}\right): X \rightarrow \mathbb{R}$ is concave (affine) on $\operatorname{dom} \mathcal{A}$, i.e.,

$$
\begin{aligned}
s_{\mathcal{A}}\left(\alpha x_{1}+(1-\alpha) x_{2}, y^{*}\right) & \geq \alpha s_{\mathcal{A}}\left(x_{1}, y^{*}\right)+(1-\alpha) s_{\mathcal{A}}\left(x_{2}, y^{*}\right) \\
\left(s_{\mathcal{A}}\left(\alpha x_{1}+(1-\alpha) x_{2}, y^{*}\right)\right. & =\alpha s_{\mathcal{A}}\left(x_{1}, y^{*}\right)+(1-\alpha) s_{\mathcal{A}}\left(x_{2}, y^{*}\right)
\end{aligned}
$$

for all $x_{1}, x_{2} \in \operatorname{dom} \mathcal{A}$ and all $\alpha \in[0,1]$.

Remark 3.3 Let $\mathcal{A}: X \rightarrow$ bcc $Y$ be an affine multifunction and $(u, v)$ a point of $X \times Y$. It is easy to see that the multifunction $\mathcal{A}_{1}: X \rightarrow \operatorname{bcc} Y$ defined by gr $\mathcal{A}_{1}=\operatorname{gr} \mathcal{A}+(u, v)$ is affine too. It shows that without loss of generality we can assume that $0 \in \operatorname{dom} \mathcal{A}$.

Remark 3.4 Let $\mathcal{A}: X \rightarrow \operatorname{bcc} Y$ be an affine multifunction with $0 \in \operatorname{dom} \mathcal{A}$ and let $U$ denote the linear hull of $\operatorname{dom} \mathcal{A}$. In the case int $(\operatorname{dom} \mathcal{A})=\emptyset$ we may define the multifunction $\mathcal{A}_{1}: X \rightarrow$ bcc $Y$ by setting $\mathcal{A}_{1}(x)=\mathcal{A}(P x)$ for all $x \in X$ where $P: X \rightarrow U$ denotes a projector of $X$ onto $U$.

Immediately from the definition of $\mathcal{A}_{1}$ we obtain $\operatorname{dom} \mathcal{A}_{1}=\operatorname{dom} \mathcal{A}+\operatorname{ker} P$, where ker $P=\{x \in X \mid P(x)=0\}$ denotes the kernel of the projector $P$. Since $\operatorname{dom} \mathcal{A}$ is a convex subset of $X$ and $\operatorname{dim} X<+\infty$ then $\operatorname{ri}(\operatorname{dom} \mathcal{A}) \neq \varnothing$ and, hence, $\operatorname{int}\left(\operatorname{dom} \mathcal{A}_{1}\right) \neq \emptyset$. Besides, it is not hard to see that $\mathcal{A}_{1}$ is an affine multifunction and $\mathcal{A}_{1}(x)=\mathcal{A}(x)$ for $x \in \operatorname{dom} \mathcal{A}$. Thus each affine multifunction $\mathcal{A}: X \rightarrow \operatorname{bcc} Y$ can be extended with preserving affinity to the effective domain whose interior is nonempty.

From the above remarks it follows that without loss of generality we can assume in what follows that affine multifunctions under consideration are such that $\operatorname{int}(\operatorname{dom} \mathcal{A}) \neq \varnothing$ and $0 \in \operatorname{int}(\operatorname{dom} \mathcal{A})$. Moreover, we can suppose that $(0,0) \in \operatorname{gr} \mathcal{A}$. 


\subsection{A conjugate mapping to an affine multifunction}

Let $\mathcal{A}: X \rightarrow \operatorname{bcc} Y$ be an affine multifunction such that $\operatorname{int}(\operatorname{dom} \mathcal{A}) \neq \varnothing$ and let $s_{\mathcal{A}}(\cdot, \cdot): X \times Y^{*} \rightarrow \mathbb{R}$ be the support function of $\mathcal{A}$. From the characterization of affine multifunctions given in Proposition 3.2, we conclude that for each $y^{*} \in Y^{*}$ the real-valued function $s_{\mathcal{A}}\left(\cdot, y^{*}\right): \operatorname{dom} \mathcal{A} \rightarrow \mathbb{R}$ is affine on $\operatorname{dom} \mathcal{A}$. Since $\operatorname{int}(\operatorname{dom} \mathcal{A}) \neq \emptyset$ then the function $s_{\mathcal{A}}\left(\cdot, y^{*}\right): \operatorname{dom} \mathcal{A} \rightarrow \mathbb{R}$ can be extended in the unique way to the entire space $X$ with preserving affinity. Consequently, with each $y^{*} \in Y^{*}$ we may associate a linear function $\mathcal{A}^{*}\left(y^{*}\right) \in X^{*}$ such that

$$
s_{\mathcal{A}}\left(x, y^{*}\right)=s_{\mathcal{A}}\left(x^{0}, y^{*}\right)+\left\langle x-x^{0}, \mathcal{A}^{*}\left(y^{*}\right)\right\rangle \text { for all } x \in \operatorname{dom} \mathcal{A} \text {. }
$$

Here $x^{0}$ is an arbitrary fixed point of $\operatorname{dom} \mathcal{A}$.

Thus, each affine multifunction $\mathcal{A}: X \rightarrow$ bcc $Y$ defines the single-valued mapping $\mathcal{A}^{*}: Y^{*} \rightarrow X^{*}$ which we call the conjugate mapping to the affine multifunction $\mathcal{A}: X \rightarrow$ bec $Y$.

It is worth emphasizing that the conjugate mapping $\mathcal{A}^{*}: Y^{*} \rightarrow X^{*}$ is single-valued while the initial mapping $\mathcal{A}: X \rightarrow Y$ is multi-valued.

Example 3.5 Let $A: X \rightarrow Y$ be a single-valued linear operator from $X$ into $Y$ and let $Q$ be a compact convex subset of $Y$. It is not hard to see that the multifunction $\mathcal{A}: x \rightarrow A x+Q$ is affine and its conjugate mapping $\mathcal{A}^{*}: Y^{*} \rightarrow X^{*}$ coincides with the linear operator $A^{*}: Y^{*} \rightarrow X^{*}$ that is conjugate to the linear operator $A: X \rightarrow Y$ in common sense for linear (single-valued) operators (cf., for instance, [39]).

Proposition 3.6 The conjugate mapping $\mathcal{A}^{*}: Y^{*} \rightarrow X^{*}$ to an affine multifunction $\mathcal{A}: X \rightarrow \operatorname{bcc} Y$ is a difference-sublinear mapping, i.e., it is positive homogeneous, continuous and for every $h \in X$ the real-valued function $y^{*} \rightarrow\left\langle h, \mathcal{A}^{*}\left(y^{*}\right)\right\rangle$ can be presented as a difference of two sublinear functions.

Moreover, the function $y^{*} \rightarrow\left\langle h, \mathcal{A}^{*}\left(y^{*}\right)\right\rangle$ is sublinear for every $h \in X$ holding $x^{0}+$ th $\in \operatorname{dom} \mathcal{A}$ for some $x^{0} \in \operatorname{dom} \mathcal{A}$ and for all $t \geq 0$.

At last, the function $y^{*} \rightarrow\left\langle h, \mathcal{A}^{*}\left(y^{*}\right)\right\rangle$ is linear whenever $h \in X$ holds $x^{0}+t h \in \operatorname{dom} \mathcal{A}$ for some $x^{0} \in \operatorname{dom} \mathcal{A}$ and for all $t \in \mathbb{R}$.

Proof Let $h \in X$. Choose a point $x^{0} \in \operatorname{int}(\operatorname{dom} \mathcal{A})$ and a real $\delta>0$ such that $x^{0}+t h \in \operatorname{dom} \mathcal{A}$ for all $t \in(0, \delta)$. Then it follows immediately from the equality (3) that for all $t \in(0, \delta)$ we have

$$
\left\langle h, \mathcal{A}^{*}\left(y^{*}\right)\right\rangle=t^{-1}\left(s_{\mathcal{A}}\left(x^{0}+t h, y^{*}\right)-s_{\mathcal{A}}\left(x^{0}, y^{*}\right)\right) .
$$

This equality shows that the function $y^{*} \rightarrow\left\langle h, \mathcal{A}^{*}\left(y^{*}\right)\right\rangle$ is difference-sublinear.

Suppose that a vector $h \in X$ holds $x^{0}+t h \in \operatorname{dom} \mathcal{A}$ for some $x^{0} \in \operatorname{dom} \mathcal{A}$ and for all $t \geq 0$. Since for each $x \in \operatorname{dom} \mathcal{A}$ the function $y^{*} \rightarrow s_{\mathcal{A}}\left(x, y^{*}\right)$ is subadditive, we have

$$
s_{\mathcal{A}}\left(x, y_{1}^{*}+y_{2}^{*}\right)-s_{\mathcal{A}}\left(x, y_{1}^{*}\right)-s_{\mathcal{A}}\left(x, y_{2}^{*}\right) \leq 0 \text { for all } y_{1}^{*}, y_{2}^{*} \in Y^{*} .
$$


Setting $x=x^{0}+t h$ we obtain from the inequality (4) and the equality (3) that

$$
\begin{aligned}
& s_{\mathcal{A}}\left(x^{0}, y_{1}^{*}+y_{2}^{*}\right)-s_{\mathcal{A}}\left(x^{0}, y_{1}^{*}\right)-s_{\mathcal{A}}\left(x^{0}, y_{2}^{*}\right) \\
& \quad+t\left[\left\langle h, \mathcal{A}^{*}\left(y_{1}^{*}+y_{2}^{*}\right)\right\rangle-\left\langle h, \mathcal{A}^{*}\left(y_{1}^{*}\right)\right\rangle-\left\langle h, \mathcal{A}^{*}\left(y_{2}^{*}\right)\right\rangle\right] \leq 0
\end{aligned}
$$

holds for all $t \geq 0$. It implies

$$
\left\langle h, \mathcal{A}^{*}\left(y_{1}^{*}+y_{2}^{*}\right)\right\rangle-\left\langle h, \mathcal{A}^{*}\left(y_{1}^{*}\right)\right\rangle-\left\langle h, \mathcal{A}^{*}\left(y_{2}^{*}\right)\right\rangle \leq 0 .
$$

Thus, the function $y^{*} \rightarrow\left\langle h, \mathcal{A}^{*}\left(y^{*}\right)\right\rangle$ is subadditive and, hence, it is sublinear.

It proves the second statement of the proposition.

Let a vector $h \in X$ holds $x^{0}+t h \in \operatorname{dom} \mathcal{A}$ for some $x^{0} \in \operatorname{dom} \mathcal{A}$ and for all $t \in \mathbb{R}$. In this case we have $x^{0}+t h \in \operatorname{dom} \mathcal{A}$ and $x^{0}+t(-h) \in \operatorname{dom} \mathcal{A}$ for all $t \geq 0$ therefore both functions $y^{*} \rightarrow\left\langle h, \mathcal{A}^{*}\left(y^{*}\right)\right\rangle$ and $y^{*} \rightarrow-\left\langle h, \mathcal{A}^{*}\left(y^{*}\right)\right\rangle$ are sublinear and, consequently, they are linear.

It completes the proof of the proposition.

Proposition 3.7 For every difference-sublinear mapping $P: Y^{*} \rightarrow X^{*}$ there exists an affine multifunction $\mathcal{A}: X \rightarrow$ bcc $Y$ such that $\mathcal{A}^{*}=P$, where $\mathcal{A}^{*}: Y^{*} \rightarrow X^{*}$ is the mapping conjugate to $\mathcal{A}$.

Proof Let $\left\{e_{1}, e_{2}, \ldots, e_{m}\right\}$ be a basis of $X$. Since the functions $p_{i}(\cdot): y^{*} \rightarrow\left\langle e_{i}, P\left(y^{*}\right)\right\rangle$, $i=1,2, \ldots, m$, are difference-sublinear we can represent each of them in the form $p_{i}(\cdot)=\underline{p}_{i}(\cdot)-\bar{p}_{i}(\cdot), i=1,2, \ldots, m$, where the functions $\underline{p}_{i}(\cdot), \bar{p}_{i}(\cdot), i=1,2, \ldots, m$ are sublinear.

Let us consider the function $q(\cdot): y^{*} \rightarrow \Sigma_{i=1}^{m}\left(p_{i}\left(y^{*}\right)+\bar{p}_{i}\left(y^{*}\right)\right)$. It is evident that $q(\cdot)$ is sublinear. Besides, for each $y^{*} \in Y^{*}$ we have

$$
q\left(y^{*}\right)+\left\langle x, P\left(y^{*}\right)\right\rangle=\sum_{i=1}^{m}\left[\left(1+x_{i}\right) \underline{p}_{i}\left(y^{*}\right)+\left(1-x_{i}\right) \bar{p}_{i}\left(y^{*}\right)\right],
$$

where $x_{1}, x_{2}, \ldots, x_{m}$ are the coordinates of the vector $x$ in the basis $\left\{e_{1}, e_{2}, \ldots, e_{m}\right\}$.

It shows that the function $y^{*} \rightarrow q\left(y^{*}\right)+\left\langle x, P\left(y^{*}\right)\right\rangle$ is also sublinear for all $x \in X$ such that $x=\sum_{i-1}^{m} x_{i} e_{i}$ with $\left|x_{i}\right| \leq 1, i=1,2, \ldots, m$.

Now let $\mathcal{A}: X \rightarrow \operatorname{bcc} Y$ be the multifunction such that $\operatorname{dom} \mathcal{A}:=\{x=$ $\left.\sum_{i=1}^{m} x_{i} e_{i}\left|x_{i}\right| \leq 1, \quad i=1,2, \ldots, m\right\}$ and $s_{\mathcal{A}}\left(x, y^{*}\right)=q\left(y^{*}\right)+\left\langle x, P\left(y^{*}\right)\right\rangle$. It follows from Proposition 3.2 that $\mathcal{A}$ is affine and, moreover, we can readily see from the definition of the conjugate mapping that $\mathcal{A}^{*}=P$. The proof is complete.

Example $3.8[20,37]$ Consider the multifunction $\mathcal{A}: \mathbb{R} \rightarrow$ bcc $\mathbb{R}^{2}$ the graph of which is given by

$$
\operatorname{gr} \mathcal{A}:=\left\{(x, y) \in \mathbb{R} \times \mathbb{R}^{2}|| y_{1}|\leq 1-x,| y_{2} \mid \leq 1+x\right\} .
$$

It is evident that $\operatorname{dom} \mathcal{A}=[-1,1]$. Using straightforward calculation we get that the support function of $\mathcal{A}$ can be presented in the form

$$
s_{\mathcal{A}}\left(x, y^{*}\right)=\left(\left|y_{1}^{*}\right|+\left|y_{2}^{*}\right|\right)+x\left(-\left|y_{1}^{*}\right|+\left|y_{2}^{*}\right|\right) .
$$


It shows that $\mathcal{A}$ is an affine multifunction and its conjugate mapping is defined by

$$
\mathcal{A}^{*}:\left(y_{1}^{*}, y_{2}^{*}\right) \rightarrow-\left|y_{1}^{*}\right|+\left|y_{2}^{*}\right| \in \mathbb{R} .
$$

Proposition 3.9 (cf. with Le Van Hot [40]) Any convex multifunction $\mathcal{A}: X \rightarrow$ bcc $Y$ with $\operatorname{dom} \mathcal{A}=X$ is actually affine and can be presented in the form

$$
\mathcal{A}(x)=A x+\mathcal{A}(0) \text { for all } x \in X,
$$

where $A: X \rightarrow Y$ is a uniquely defined single-valued linear operator from $X$ to $Y$.

Proof Observe that $s_{\mathcal{A}}\left(x,-y^{*}\right)=-\min _{y \in \mathcal{A}(x)}\left\langle y, y^{*}\right\rangle$ for all $y^{*} \in Y^{*}$ and $x \in X$.

It shows that for each $y^{*} \in Y^{*}$ the concave function $x \rightarrow s_{\mathcal{A}}\left(x, y^{*}\right)$ majorizes the convex function $x \rightarrow-s_{\mathcal{A}}\left(x,-y^{*}\right)$, i.e., for each $y^{*} \in Y^{*}$

$$
-s_{\mathcal{A}}\left(x,-y^{*}\right) \leq s_{\mathcal{A}}\left(x, y^{*}\right) \text { for all } x \in X .
$$

Fix $y^{*} \in Y^{*}$ and let $x_{1}^{*} \in X^{*}$ be an arbitrary subgradient of the concave function $x \rightarrow s_{\mathcal{A}}\left(x, y^{*}\right)$ at a point $x_{1} \in X$ and let $x_{2}^{*} \in X^{*}$ be an arbitrary subgradient of the convex function $x \rightarrow-s_{\mathcal{A}}\left(x,-y^{*}\right)$ at a point $x_{2} \in X$. Then we have

$$
\begin{gathered}
-s_{\mathcal{A}}\left(x_{1},-y^{*}\right)+\left\langle x_{1}^{*}, x-x_{1}\right\rangle \leq-s_{\mathcal{A}}\left(x,-y^{*}\right) \\
\leq s_{\mathcal{A}}\left(x, y^{*}\right) \leq s_{\mathcal{A}}\left(x_{2}^{*}, y^{*}\right)+\left\langle x_{2}^{*}, x-x_{2}\right\rangle
\end{gathered}
$$

for all $x \in X$. From this inequality we deduce that the real-valued affine function

$$
u: x \rightarrow\left\langle x_{2}^{*}-x_{1}^{*}, x\right\rangle+s_{\mathcal{A}}\left(x_{2}, y^{*}\right)+s_{\mathcal{A}}\left(x_{1},-y^{*}\right)+\left\langle x_{1}^{*}, x_{1}\right\rangle-\left\langle x_{2}^{*}, x_{2}\right\rangle
$$

is nonnegative on $X$. But it is possible if and only if the function $u$ is constant or, equivalently, if and only if $x_{1}^{*}=x_{2}^{*}$.

It shows that at each point $x \in X$ the concave function $x \rightarrow s_{\mathcal{A}}\left(x, y^{*}\right)$ has the only subgradient and, moreover, this subgradient does not depend on $x$. It implies that the function $x \rightarrow s_{\mathcal{A}}\left(x, y^{*}\right)$ is affine in $x$. By Proposition 3.2 we conclude that $\mathcal{A}$ is an affine multifunction.

Since $\mathcal{A}: X \rightarrow \operatorname{bcc} Y$ is an affine multifunction with $\operatorname{dom} \mathcal{A}=X$ then its conjugate mapping $\mathcal{A}^{*}: Y^{*} \rightarrow X^{*}$ satisfies the equality

$$
s_{\mathcal{A}}\left(x, y^{*}\right)=s_{\mathcal{A}}\left(0, y^{*}\right)+\left\langle x, \mathcal{A}^{*}\left(y^{*}\right)\right\rangle
$$

for all $x \in X$ and $y^{*} \in Y^{*}$. Moreover, it follows from the last statement of Proposition 3.6 that the function $y^{*} \rightarrow\left\langle x, \mathcal{A}^{*}\left(y^{*}\right)\right\rangle$ is linear for each $y^{*} \in Y^{*}$. Consequently, $\mathcal{A}^{*}: Y^{*} \rightarrow X^{*}$ is a single-valued linear operator from $Y^{*}$ into $X^{*}$. Now, let $A: X \rightarrow Y$ be the linear operator conjugate to $\mathcal{A}^{*}: Y^{*} \rightarrow X^{*}$ in the sense of the theory of linear operators, i.e.,

$$
\left\langle x, \mathcal{A}^{*}\left(y^{*}\right)\right\rangle=\left\langle A x, y^{*}\right\rangle \text { for all } y^{*} \in Y^{*} \text { and all } x \in X \text {. }
$$


Then we have from (5) that

$$
s_{\mathcal{A}}\left(x, y^{*}\right)=s_{\mathcal{A}}\left(0, y^{*}\right)+\left\langle A x, y^{*}\right\rangle \quad \text { for all } y^{*} \in Y^{*} \text { and all } x \in X
$$

and we conclude that

$$
\mathcal{A}(x)=A x+\mathcal{A}(0) \text { for all } x \in X .
$$

It completes the proof of the proposition.

\subsection{Linear processes associated with affine multifunctions}

A multifunction $\mathcal{R}: X \rightarrow$ bcc $Y$ will be called a linear process if it is

(i) positive homogeneous:

$$
\mathcal{R}(t x)=t \mathcal{R}(x) \text { for all } x \in \operatorname{dom} \mathcal{R} \text { and all } t \geq 0 ;
$$

(ii) additive:

$$
\mathcal{R}\left(x_{1}+x_{2}\right)=\mathcal{R}\left(x_{1}\right)+\mathcal{R}\left(x_{2}\right) \text { for all } x_{1}, x_{2} \in \operatorname{dom} \mathcal{R} .
$$

It is evident that dom $\mathcal{R}$ and gr $\mathcal{R}$ are convex cones in $X$ and in $X \times Y$ respectively, and $\mathcal{R}(0)=\{0\}$. Besides, it is immediate consequence of the above definition that any linear process $\mathcal{R}: X \rightarrow$ bcc $Y$ is an affine multifunction.

Let $\mathcal{R}^{*}: Y^{*} \rightarrow X^{*}$ be the conjugate mapping to a linear process $\mathcal{R}: X \rightarrow$ bcc $Y$. Since $\operatorname{dom} \mathcal{R}$ is a convex cone then due to Proposition 3.6, for each $x \in \operatorname{dom} \mathcal{R}$ the function $y^{*} \rightarrow\left\langle x, \mathcal{R}^{*}\left(y^{*}\right)\right\rangle$ is sublinear.

Notice that $0 \in \operatorname{dom} \mathcal{R}$ and $s_{\mathcal{R}}\left(0, y^{*}\right) \equiv 0$ for all $y^{*} \in Y^{*}$. Hence it follows from the definition of the conjugate mapping that

$$
s_{\mathcal{R}}\left(x, y^{*}\right)=\left\langle x, \mathcal{R}^{*}\left(y^{*}\right)\right\rangle \text { for all } x \in \operatorname{dom} \mathcal{R} \text { and all } y^{*} \in Y^{*}
$$

and, consequently, for each $x \in \operatorname{dom} \mathcal{R}$ we have

$$
\mathcal{R}(x)=\left\{y \in Y \mid\left\langle y, y^{*}\right\rangle \leq\left\langle x, \mathcal{R}^{*}\left(y^{*}\right)\right\rangle \text { for all } y^{*} \in Y\right\} .
$$

Proposition 3.10 Let $\mathcal{A}: X \rightarrow \operatorname{bcc} Y$ be an affine multifunction. Then there exists a linear process $\mathcal{R}: X \rightarrow \operatorname{bcc} Y$ with $\operatorname{dom} \mathcal{R}=0^{+}(\operatorname{dom} \mathcal{A})$ and

$$
\mathcal{A}(x+h)=\mathcal{A}(x)+\mathcal{R}(h)
$$

for all $x \in \operatorname{dom} \mathcal{A}$ and all $h \in 0^{+}(\operatorname{dom} \mathcal{A})$.

Here $0^{+}(\operatorname{dom} \mathcal{A})$ is the recession cone of $\operatorname{dom} \mathcal{A}$.

Proof Let $\mathcal{A}^{*}: Y^{*} \rightarrow X^{*}$ be the conjugate mapping to $\mathcal{A}$. Setting $\operatorname{dom} \mathcal{R}=0^{+}(\operatorname{dom} \mathcal{A})$ and $\mathcal{R}(x)=\left\{y \in Y \mid\left\langle y, y^{*}\right\rangle \leq\left\langle x, \mathcal{A}^{*}\left(y^{*}\right)\right\rangle\right\}$ for all $x \in \operatorname{dom} \mathcal{R}$ we define the multifunction $\mathcal{R}$. It is evident that $\mathcal{R}: X \rightarrow$ bcc $Y$ is a linear process and

$$
s_{\mathcal{A}}\left(x+h, y^{*}\right)=s_{\mathcal{A}}\left(x, y^{*}\right)+s_{\mathcal{R}}\left(h, y^{*}\right)
$$

for all $x \in \operatorname{dom} \mathcal{A}, h \in 0^{+}(\operatorname{dom} \mathcal{A})$ and all $y^{*} \in Y^{*}$. The last equality is equivalent to (6).

The proposition is proved. 
Remark 3.11 A multifunction $\mathcal{R}: X \rightarrow$ bec $Y$ is a linear process if and only if it is both a convex process $[30,41,42]$ and a fan $[32,43]$. We refer readers to papers and monographs mentioned above for more detail accounts on convex processes and fans.

\subsection{Some other properties of affine multifunctions}

Let us recall (see, for instance, [5]) that a multifunction $F: X \rightarrow$ bcc $Y$ is Lipschitzian on a set $Q \subset \operatorname{dom} \mathcal{A}$ if there exists a real number $L_{Q}$ such that for all $x_{1}, x_{2} \in Q$ we have

$$
F\left(x_{1}\right) \subset F\left(x_{2}\right)+L_{Q}\left\|x_{1}-x_{2}\right\| B_{Y} .
$$

Here $B_{Y}$ is a unit ball of $Y$.

The real $L_{Q}$ is called the Lipschitz constant for $F$ on $Q$.

Proposition 3.12 An affine multifunction $\mathcal{A}: X \rightarrow$ bcc $Y$ is Lipschitzian on its effective domain $\operatorname{dom} \mathcal{A}$ with Lipschitz constant $L=\left\|\mathcal{A}^{*}\right\|$, where

$$
\left\|\mathcal{A}^{*}\right\|=\max _{\left\|y^{*}\right\| \leq 1}\left\|\mathcal{A}^{*}\left(y^{*}\right)\right\|
$$

Proof From positive homogeneity and continuity (it is sufficient continuity at zero) of the conjugate mapping $\mathcal{A}^{*}: Y^{*} \rightarrow X^{*}$ we conclude that there exists $L \geq 0$ such that

$$
\left\|\mathcal{A}^{*}\left(y^{*}\right)\right\| \leq L\left\|y^{*}\right\| \quad \text { for all } y^{*} \in Y^{*},
$$

with the smallest constant $L$ for which (6) holds being equal to $\left\|\mathcal{A}^{*}\right\|$.

Now from (4) and (6) we obtain that for all $x_{1}, x_{2} \in \operatorname{dom} \mathcal{A}$ and all $y^{*} \in Y^{*}$

$$
\begin{aligned}
& \left|s_{\mathcal{A}}\left(x_{1}, y^{*}\right)-s_{\mathcal{A}}\left(x_{2}, y^{*}\right)\right|=\left|\left\langle x_{1}-x_{2}, \mathcal{A}^{*}\left(y^{*}\right)\right\rangle\right| \\
& \leq\left\|\mathcal{A}^{*}\left(y^{*}\right)\right\|\left\|x_{1}-x_{2}\right\| \leq\left\|\mathcal{A}^{*}\right\|\left\|y^{*}\right\|\left\|x_{1}-x_{2}\right\|
\end{aligned}
$$

and, consequently,

$$
s_{\mathcal{A}}\left(x_{1}, y^{*}\right) \leq s_{\mathcal{A}}\left(x_{2}, y^{*}\right)+\left\|\mathcal{A}^{*}\right\|\left\|x_{1}-x_{2}\right\|\left\|y^{*}\right\|
$$

for all $x_{1}, x_{2} \in \operatorname{dom} \mathcal{A}$ and all $y^{*} \in Y^{*}$.

Since the function $y^{*} \rightarrow\left\|y^{*}\right\|$ is the support function of the unit ball $B_{Y} \subset Y$ the last inequality is equivalent to the inclusion

$$
\mathcal{A}\left(x_{1}\right) \subset \mathcal{A}\left(x_{2}\right)+\left\|\mathcal{A}^{*}\right\|\left\|x_{1}-x_{2}\right\| B_{Y} \quad \text { for all } x_{1}, x_{2} \in \operatorname{dom} \mathcal{A} .
$$

The proposition is proved.

Proposition 3.13 An affine multifunction $\mathcal{A}: X \rightarrow$ bcc $Y$ is uniformly continuous in the Hausdorff sense on $\operatorname{dom} \mathcal{A}$. 
Proof Using the equality

$$
d_{H}\left(\mathcal{A}\left(x_{1}\right), \mathcal{A}\left(x_{2}\right)\right)=\max _{\left\|y^{*}\right\| \leq 1}\left|s_{\mathcal{A}}\left(x_{1}, y^{*}\right)-s_{\mathcal{A}}\left(x_{2}, y^{*}\right)\right|
$$

we obtain from inequality (8) that

$$
d_{H}\left(\mathcal{A}\left(x_{1}\right), \mathcal{A}\left(x_{2}\right)\right) \leq\left\|\mathcal{A}^{*}\right\|\left\|x_{1}-x_{2}\right\| \quad \text { for all } x_{1}, x_{2} \in \operatorname{dom} \mathcal{A}
$$

and it completes the proof of the proposition.

\subsection{Inextensible affine multifunctions}

We say that an affine multifunction $\mathcal{A}: X \rightarrow \operatorname{bcc} Y$ is inextensible if there is no affine extension of $\mathcal{A}$ other than $\mathcal{A}$ itself.

It is an immediate consequence of Remark 3.4 that an effective domain of any inextensible affine multifunction has a nonempty interior.

Proposition 3.14 For any affine multifunction $\mathcal{A}: X \rightarrow \operatorname{bcc} Y$ with $\operatorname{int}(\operatorname{dom} \mathcal{A}) \neq \varnothing$ there exists the uniquely defined inextensible affine extension of $\mathcal{A}$.

Proof Let $\mathcal{A}^{*}: Y^{*} \rightarrow X^{*}$ be the mapping conjugate to $\mathcal{A}$. Fix a point $x^{0} \in \operatorname{int}(\operatorname{dom} \mathcal{A})$ and consider the real-valued function $\hat{s}_{\mathcal{A}}: X \times Y^{*} \rightarrow \mathbb{R}$ defined by

$$
\left(x, y^{*}\right) \rightarrow \hat{s}_{\mathcal{A}}\left(x, y^{*}\right):=s_{\mathcal{A}}\left(x^{0}, y^{*}\right)+\left\langle x-x^{0}, \mathcal{A}^{*}\left(y^{*}\right)\right\rangle .
$$

Straightforward from the definition of $\mathcal{A}^{*}$ it follows that for each $y^{*} \in Y^{*}$ the function $\hat{s}_{\mathcal{A}}\left(\cdot, y^{*}\right): X \rightarrow \mathbb{R}$ is the uniquely defined affine extension of the function $s_{\mathcal{A}}\left(\cdot, y^{*}\right): \operatorname{dom} \mathcal{A} \rightarrow \mathbb{R}$ to the entire space $X$. Using Proposition 3.6 we conclude that for each $x \in X$ the function $\hat{s}_{\mathcal{A}}(x, \cdot): Y^{*} \rightarrow \mathbb{R}$ is difference-sublinear and, hence, it is positive homogeneous and continuous.

Consider the multifunction $\hat{\mathcal{A}}: X \rightarrow$ bcc $Y$ such that

$$
\operatorname{dom} \widehat{\mathcal{A}}=\left\{x \in X \mid \hat{s}_{\mathcal{A}}(x, \cdot): Y^{*} \rightarrow \mathbb{R} \text { is sublinear }\right\}
$$

and the value of $\widehat{\mathcal{A}}$ at every $x \in \operatorname{dom} \widehat{\mathcal{A}}$ is defined by

$$
\widehat{\mathcal{A}}(x)=\left\{y \in Y \mid\left\langle y, y^{*}\right\rangle \leq \hat{s}_{\mathcal{A}}\left(x, y^{*}\right) \text { for all } y^{*} \in Y^{*}\right\} .
$$

It is not difficult to verify that $\widehat{\mathcal{A}}$ is an affine extension of $\mathcal{A}$.

It remains to prove that $\widehat{\mathcal{A}}$ is an inextensible affine multifunction.

Let $\mathcal{B}: X \rightarrow$ bec $Y$ be an affine extension of $\widehat{\mathcal{A}}$. Note that $\mathcal{B}$ is an affine extension of $\mathcal{A}$ as well. Hence, $s_{\mathcal{A}}\left(x, y^{*}\right)=s_{\mathcal{B}}\left(x, y^{*}\right)$ for all $x \in \operatorname{dom} \mathcal{A}$ and all $y^{*} \in Y^{*}$. Since for each $y^{*} \in Y^{*}$ there exists an only affine extension of the real-valued function $s_{\mathcal{A}}\left(\cdot, y^{*}\right): \operatorname{dom} \mathcal{A} \rightarrow \mathbb{R}$ on the entire space $X$ then $s_{\mathcal{B}}\left(x, y^{*}\right)=\hat{s}_{\mathcal{A}}\left(x, y^{*}\right)$ for all $x \in \operatorname{dom} \mathcal{B}$ and all $y^{*} \in Y^{*}$. It implies that $\operatorname{dom} \mathcal{B} \subset \operatorname{dom} \widehat{\mathcal{A}}$ and it proves that the affine multifunction $\widehat{\mathcal{A}}: X \rightarrow$ bcc $Y$ is inextensible.

The proof is complete. 
Remark 3.15 In fact in the proof of Proposition 3.14 we showed the existence of the duality between the collection of all inextensible affine multifunctions from $X$ into $Y$ and the collection of real-valued functions $s(\cdot, \cdot): X \times Y^{*} \rightarrow \mathbb{R}$ satisfying the following conditions:

(a) for each $y^{*} \in Y^{*}$ the partial function $s\left(\cdot, y^{*}\right): X \rightarrow \mathbb{R}$ is affine on $X$;

(b) for each $x \in X$ the partial function $s(x, \cdot): Y^{*} \rightarrow \mathbb{R}$ is difference-sublinear on $Y^{*}$;

(c) the set $\left\{x \in X \mid s(x, \cdot): Y^{*} \rightarrow \mathbb{R}\right.$ is sublinear $\}$ has a nonempty interior.

Remark 3.16 Let $\mathcal{A}_{1}: X \rightarrow$ bcc $Y$ and $\mathcal{A}_{2}: X \rightarrow$ bcc $Y$ be two inextensible affine multifunctions. If there exists an open set $U \subset \operatorname{dom} \mathcal{A}_{1} \cap \operatorname{dom} \mathcal{A}_{2}$ such that $\mathcal{A}_{1}(x)=\mathcal{A}_{2}(x)$ for all $x \in U$ then $\mathcal{A}_{1}(x)=\mathcal{A}_{2}(x)$ for all $x \in X$ or, equivalently, $\operatorname{gr} \mathcal{A}_{1}=\operatorname{gr} \mathcal{A}_{2}$.

Proposition 3.17 For each inextensible affine multifunction $\mathcal{A}: X \rightarrow$ bcc $Y$ its effective domain $\operatorname{dom} \mathcal{A}$ is a closed subset of $X$.

Proof Let $x \in \operatorname{cl}(\operatorname{dom} \mathcal{A})$ and $x^{0} \in \operatorname{int}(\operatorname{dom} \mathcal{A})$. Then $x_{t}:=t x+(1-t) x^{0} \in \operatorname{int}(\operatorname{dom} \mathcal{A})$ for all $t \in[0,1)$. Hence, for all $y_{1}^{*}, y_{2}^{*} \in Y^{*}$ and $t \in[0,1)$, we have

$$
\hat{s}_{\mathcal{A}}\left(x_{t}, y_{1}^{*}+y_{2}^{*}\right) \leq \hat{s}_{\mathcal{A}}\left(x_{t}, y_{1}^{*}\right)+\hat{s}_{\mathcal{A}}\left(x_{t}, y_{2}^{*}\right) .
$$

Here $\hat{s}_{\mathcal{A}}\left(\cdot, y^{*}\right): X \rightarrow \mathbb{R}$ is the affine extension of the function $s_{\mathcal{A}}\left(\cdot, y^{*}\right): \operatorname{dom} \mathcal{A} \rightarrow \mathbb{R}$ to the entire space $X$.

Since for each $y^{*} \in Y^{*}$ the function $\hat{s}_{\mathcal{A}}\left(\cdot, y^{*}\right): X \rightarrow \mathbb{R}$ is affine and, consequently, continuous then tending $t$ to 1 in the last inequality we obtain

$$
\hat{s}_{\mathcal{A}}\left(x, y_{1}^{*}+y_{2}^{*}\right) \leq \hat{s}_{\mathcal{A}}\left(x, y_{1}^{*}\right)+\hat{s}_{\mathcal{A}}\left(x, y_{2}^{*}\right)
$$

Due to inextensibility of $\mathcal{A}$ we deduce from the latter inequality that $x \in \operatorname{dom} \mathcal{A}$. It completes the proof.

Proposition 3.18 Each affine multifunction $\mathcal{A}: X \rightarrow$ bcc $Y$ with a closed effective domain $\operatorname{dom} \mathcal{A}$ is closed. In particular, an inextensible affine multifunction $\mathcal{A}$ is closed.

Proof The graph of an affine multifunction $\mathcal{A}: X \rightarrow$ bcc $Y$ can be presented in the form

$$
\operatorname{gr} \mathcal{A}=\bigcap_{y^{*} \in Y^{*}}\left\{(x, y) \in \operatorname{dom} \mathcal{A} \times Y \mid\left\langle y, y^{*}\right\rangle-\left\langle x-x^{0}, \mathcal{A}^{*}\left(y^{*}\right)\right\rangle \leq s_{\mathcal{A}}\left(x^{0}, y^{*}\right)\right\}
$$

where $x^{0}$ is a fixed point of $\operatorname{dom} \mathcal{A}$. It shows that in the case, when $\operatorname{dom} \mathcal{A}$ is closed, the graph gr $\mathcal{A}$ is the intersection of the family of closed sets. The second assertion follows from Proposition 3.17.

Example 3.19 Let $\mathcal{A}: \mathbb{R} \rightarrow$ bcc $\mathbb{R}^{2}$ be the affine multifunction given in Example 3.8. Recall that $\operatorname{dom} \mathcal{A}=[-1,1]$. Representing the support function of $\mathcal{A}$ in the form

$$
s_{\mathcal{A}}\left(x, y^{*}\right)=(1-x)\left|y_{1}^{*}\right|+(1+x)\left|y_{2}^{*}\right|
$$


we see that it is sublinear in $y^{*}$ only for $x \in[-1,1]$. It shows that $\mathcal{A}$ is an inextensible affine multifunction.

\section{Differentiability of multifunctions}

\subsection{Definition of Fréchet differentiability}

Let $X$ and $Y$ be finite-dimensional normed vector spaces over $\mathbb{R}$ and $F: X \rightarrow$ bcc $Y$ a multifunction from $X$ into $Y$ with $\operatorname{int}(\operatorname{dom} F) \neq \emptyset$.

Definition 4.1 We say that a multifunction $F: X \rightarrow$ bcc $Y$ is Fréchet differentiable at a point $x^{0} \in \operatorname{int}(\operatorname{dom} F)$ if there exists an affine multifunction $\mathcal{A}\left(x^{0} \mid \cdot\right): X \rightarrow \operatorname{bcc} Y$ such that $0 \in \operatorname{int}\left(\operatorname{dom} \mathcal{A}\left(x^{0} \mid \cdot\right)\right)$ and

$$
\lim _{h \rightarrow 0} \frac{d_{H}\left(F\left(x^{0}+h\right), \mathcal{A}\left(x^{0} \mid h\right)\right)}{\|h\|}=0 .
$$

Here $d_{H}(\cdot, \cdot)$ stands for the Hausdorff metric on bcc $Y$.

It is easy to see that $\mathcal{A}\left(x^{0} \mid 0\right)=F\left(x^{0}\right)$.

Without loss of generality we can assume that an affine multifunction $\mathcal{A}\left(x^{0} \mid \cdot\right)$ satisfying Definition 4.1 is inextensible. In this case such a multifunction is uniquely determined by (10). To show it we observe that equality (10) is equivalent to the following statement: for any $\varepsilon>0$ there exists $\delta>0$ such that for all $h \in \delta B_{X}$ we have

$$
F\left(x^{0}+h\right) \subset \mathcal{A}\left(x^{0} \mid h\right)+\varepsilon\|h\| B_{Y}
$$

and

$$
\mathcal{A}\left(x^{0} \mid h\right) \subset F\left(x^{0}+h\right)+\varepsilon\|h\| B_{Y} .
$$

Here $B_{X}$ and $B_{Y}$ stands for unit balls in $X$ and $Y$ respectively.

Now let us suppose that two inextensible affine multifunctions $\mathcal{A}_{1}\left(x^{0} \cdot \mid \cdot\right): X \rightarrow$ bcc $Y$ and $\mathcal{A}_{2}\left(x^{0} \mid \cdot\right): X \rightarrow$ bcc $Y$ satisfy Definition 4.1 . Then it follows from the above observation that for any $\varepsilon>0$ there exists $\delta>0$ such that for all $h \in \delta B_{X}$ we have

$$
\mathcal{A}_{1}\left(x^{0} \mid h\right) \subset \mathcal{A}_{2}\left(x^{0} \mid h\right)+\varepsilon\|h\| B_{Y}
$$

and

$$
\mathcal{A}_{2}\left(x^{0} \mid h\right) \subset \mathcal{A}_{1}\left(x^{0} \mid h\right)+\varepsilon\|h\| B_{Y} .
$$

Due to the Minkowski duality the last inclusions are equivalent to the following inequality

$$
\left|s_{\mathcal{A}_{1}}\left(x^{0} ; h, y^{*}\right)-s_{\mathcal{A}_{2}}\left(x^{0} ; h, y^{*}\right)\right| \leq \varepsilon\|h\|\left\|y^{*}\right\|,
$$


where $s_{\mathcal{A}}\left(x^{0} ; \cdot, \cdot\right):\left(h, y^{*}\right) \rightarrow \max _{y \in \mathcal{A}_{i}\left(x^{0} \mid h\right)}\left\langle y, y^{*}\right\rangle$ is the support function of the multifunction $\mathcal{A}_{i}\left(x^{0} \mid \cdot\right)$. Since $s_{\mathcal{A}_{i}}\left(x^{0} ; \cdot, y^{*}\right): X \rightarrow Y, i=1,2$, are affine functions and $\varepsilon$ is an arbitrary positive real number then we deduce from (13) that $s_{\mathcal{A}_{1}}\left(x^{0} ; h, y^{*}\right)=$ $s_{\mathcal{A}_{2}}\left(x^{0} ; h, y^{*}\right)$ for all $h \in \operatorname{dom} \mathcal{A}_{1} \cap \operatorname{dom} \mathcal{A}_{2}$ and all $y^{*} \in Y^{*}$. It implies that $\mathcal{A}_{1}\left(x^{0} \mid h\right)=$ $\mathcal{A}_{2}\left(x^{0} \mid h\right)$ for all $h \in \operatorname{dom} \mathcal{A}_{1} \cap \operatorname{dom} \mathcal{A}_{2}$. Thus, the inextensible affine multifunctions $\mathcal{A}_{1}$ and $\mathcal{A}_{2}$ coincide in some neighborhood of zero and, consequently, by Remark 3.16 they coincide everywhere in $X$.

The uniquely defined inextensible affine multifunction $\mathcal{A}\left(x^{0} \mid \cdot\right): X \rightarrow$ bcc $Y$ satisfying Definition 4.1 will be called the Fréchet differential approximation of the multifunction $F: X \rightarrow$ bec $Y$ at the point $x^{0}$ and will be denoted by $D F\left(x^{0} \mid \cdot\right)$.

As it follows from Proposition 3.1, the Fréchet differential approximation $D F\left(x^{0} \mid \cdot\right)$ of a multifunction $F: X \rightarrow$ bcc $Y$ is a single-valued affine function, whenever the multifunction $F$ is Fréchet differentiable at a point $x^{0} \in \operatorname{int}(\operatorname{dom} \mathcal{A})$ and $F\left(x^{0}\right)=\left\{y^{0}\right\}$ is a singleton. It shows that for a single-valued mapping $F: X \rightarrow Y$ the Fréchet differentiability of $F$ in the sense of the above definition is equivalent to the classical notion of the Fréchet differentiability for single-valued mappings. In this case we have $D F\left(x^{0} \mid h\right)=F\left(x^{0}\right)+F^{\prime}\left(x^{0}\right) h, h \in X$, where $D F\left(x^{0} \mid \cdot\right)$ is the Fréchet differential approximation of $F$ at $x^{0}$ and $F^{\prime}\left(x^{0}\right): X \rightarrow Y$ is the classical Fréchet derivative of $F$ at $x^{0}$.

Example 4.2 Let $\Omega$ be an open subset of $X$ and let $f: \Omega \rightarrow Y$ be a single-valued function, $M$ a compact convex subset of $Y$.

The multifunction $F: X \rightarrow$ bcc $Y$ defined by

$$
\operatorname{gr} F:=\{(x, y) \in X \times Y \mid x \in \Omega, y \in f(x)+M\}
$$

is Fréchet differentiable at a point $x^{0} \in \Omega$ if and only if the single-valued function $f: \Omega \rightarrow Y$ is Fréchet differentiable at $x^{0}$ and the Fréchet differential approximation of $F$ at $x^{0}$ is defined by

$$
\operatorname{gr} D F\left(x^{0} \mid \cdot\right)=\left\{(h, y) \in X \times Y \mid h \in X, y \in f\left(x^{0}\right)+f^{\prime}\left(x^{0}\right) h+M\right\} \text {. }
$$

Here $f^{\prime}\left(x^{0}\right): X \rightarrow Y$ is the classical Fréchet derivative of $f$ at $x^{0}$.

Example 4.3 Let $Y$ be endowed with a partial order $\preceq$ defined by

$$
y_{1} \preceq y_{2} \text { if and only if } y_{2}-y_{1} \in C,
$$

where $C$ is a convex closed cone with int $C \neq \emptyset$ and $C \cap(-C)=\{0\}$.

Under these assumptions an order interval $\left[y_{1}, y_{2}\right]:=\left\{y \in Y \mid y_{1} \preceq y \preceq y_{2}\right\}$ is a compact convex subset of $Y$ for each $y_{1}, y_{2} \in Y$. Without loss of generality we can also suppose that the norm given on $Y$ is such that $B_{Y}=[-e, e]$ for some $e \in \operatorname{int} C$ (in other case we can renorm the space $Y$ ).

Let $\Omega$ be an open subset of $X$ and let $f_{1}: \Omega \rightarrow Y$ and $f_{2}: \Omega \rightarrow Y$ be single-valued functions such that $f_{2}\left(x^{0}\right)-f_{1}\left(x^{0}\right) \in \operatorname{int} C$ for some $x^{0} \in \Omega$.

The multifunction $F: X \rightarrow$ bcc $Y$ defined by

$$
\operatorname{gr} F:=\left\{(x, y) \in X \times Y \mid x \in \Omega, f_{1}(x) \preceq y \preceq f_{2}(x)\right\}
$$


is Fréchet differentiable at $x^{0}$ if and only if the functions $f_{1}: \Omega \rightarrow Y$ and $f_{2}: \Omega \rightarrow Y$ are classically Fréchet differentiable at the point $x^{0}$. The Fréchet differentiable approximation of $F$ at $x^{0}$ can be defined by

$$
\begin{aligned}
\operatorname{gr} D F\left(x^{0} \mid \cdot\right)= & \{(h, y) \in X \times Y \mid h \in X, \\
& \left.f_{1}\left(x^{0}\right)+f_{1}^{\prime}\left(x^{0}\right) h \preceq y \preceq f_{2}\left(x^{0}\right)+f_{2}^{\prime}\left(x^{0}\right) h\right\},
\end{aligned}
$$

where $f_{1}^{\prime}\left(x^{0}\right): X \rightarrow Y$ and $f_{2}^{\prime}\left(x^{0}\right): X \rightarrow Y$ are the classical Fréchet derivatives of $f_{1}$ and $f_{2}$ at $x^{0}$ respectively.

Theorem 4.4 If a multifunction $F: X \rightarrow$ bcc $Y$ is Fréchet differentiable at a point $x^{0} \in \operatorname{int}(\operatorname{dom} F)$ then it is continuous in Hausdorff sense at the point $x^{0}$.

Proof Fix a positive real number $\varepsilon>0$. Since $F$ is Fréchet differentiable at the point $x^{0} \in \operatorname{int}(\operatorname{dom} F)$ then there exists $\delta>0$ such that $\delta B_{X} \subset \operatorname{dom} D F\left(x^{0} \mid \cdot\right) \cap \operatorname{dom} F$ and

$$
d_{H}\left(F\left(x^{0}+h\right), D F\left(x^{0} \mid h\right)\right) \leq \varepsilon\|h\| \text { for all } h \in \delta B_{X} .
$$

On the other side, the multifunction $D F\left(x^{0} \mid \cdot\right): X \rightarrow Y$ is Lipschitzian on $\operatorname{dom} D F\left(x^{0} \mid \cdot\right)$ and hence for some $L>0$ we have

$$
d_{H}\left(D F\left(x^{0} \mid h\right), D F\left(x^{0} \mid 0\right)\right) \leq L\|h\| \quad \text { for all } h \in \operatorname{dom} D F\left(x^{0} \mid \cdot\right) .
$$

Since $D F\left(x^{0} \mid 0\right)=F\left(x^{0}\right)$ we get from these inequalities that

$$
\begin{aligned}
& d_{H}\left(F\left(x^{0}+h\right), F\left(x^{0}\right)\right) \leq d_{H}\left(F\left(x^{0}+h\right), D F\left(x^{0} \mid h\right)\right) \\
& \quad+d_{H}\left(D F\left(x^{0} \mid h\right), D F\left(x^{0} \mid 0\right)\right) \leq(\varepsilon+L)\|h\| \text { for all } x \in \delta B_{H} .
\end{aligned}
$$

It shows that $F$ is Hausdorff continuous at $x^{0}$. The proof of the theorem is complete.

\subsection{Characterization of differentiability of multifunctions via differentiability of their support functions}

Definition $4.5[1,28] \quad$ A multifunction $F: X \rightarrow$ bcc $Y$ is called weakly differentiable at a point $x^{0} \in \operatorname{int}(\operatorname{dom} F)$ if for each $y^{*} \in Y^{*}$ the real-valued function $s_{F}\left(\cdot, y^{*}\right): \operatorname{dom} F \rightarrow \mathbb{R}$ is classically Fréchet differentiable at $x^{0}$.

It means that for each $y^{*} \in Y^{*}$ there exists a linear functional $s_{F}^{\prime}\left(x^{0}, y^{*}\right) \in X^{*}$ such that

$$
\lim _{h \rightarrow 0} \frac{s_{F}\left(x^{0}+h, y^{*}\right)-s_{F}\left(x^{0}, y^{*}\right)-\left\langle h, s_{F}^{\prime}\left(x^{0}, y^{*}\right)\right\rangle}{\|h\|}=0 .
$$

Whenever a multifunction $F: X \rightarrow$ bcc $Y$ is weakly differentiable at a point $x^{0} \in$ $\operatorname{int}(\operatorname{dom} F)$ it generates the single-valued mapping $s_{F}^{\prime}\left(x^{0}, \cdot\right): Y^{*} \ni y^{*} \rightarrow s_{F}^{\prime}\left(x^{0}, y^{*}\right) \in X^{*}$ which is called a conjugate derivative [2] of the multifunction $F$ at the point $x^{0}$. 
It follows immediately from (14) that for each $h \in X$ the function $y^{*} \rightarrow\left\langle h, s_{F}^{\prime}\left(x^{0}, y^{*}\right)\right\rangle$ is the pointwise limit of positively homogeneous functions:

$$
\left\langle h, s_{F}^{\prime}\left(x^{0}, y^{*}\right)\right\rangle=\lim _{t \rightarrow 0} \frac{s_{F}\left(x^{0}+t h, y^{*}\right)-s_{F}\left(x^{0}, y^{*}\right)}{t}=0
$$

and hence it is also positively homogeneous.

Thus the following theorem is true.

THEOREM 4.6 [2] If a multifunction $F: X \rightarrow$ bcc $Y$ is weakly differentiable at a point $x^{0} \in \operatorname{int}(\operatorname{dom} F)$ then its conjugate derivative $s_{F}^{\prime}\left(x^{0}, \cdot\right): Y^{*} \rightarrow X^{*}$ is a positively homogeneous mapping from $Y^{*}$ into $X^{*}$.

Definition 4.7 [2] A multifunction $F: X \rightarrow$ bcc $Y$ is called $R$-differentiable at a point $x^{0} \in \operatorname{int}(\operatorname{dom} F)$ if

(i) $F$ is weakly differentiable at $x^{0}$;

(ii) for any $\varepsilon>0$ there exists $\delta>0$ such that for all $h \in \delta B_{X}$ and all $y^{*} \in Y^{*}$ the inequality

$$
\left|s_{F}\left(x^{0}+h, y^{*}\right)-s_{F}\left(x^{0}, y^{*}\right)-\left\langle h, s_{F}^{\prime}\left(x^{0}, y^{*}\right)\right\rangle\right| \leq \varepsilon\|h\|\left\|y^{*}\right\|
$$

is satisfied.

The condition (ii) means that the limit in (14) is uniform with respect to $y^{*}$ on the unit ball $B_{Y^{*}}$. It implies that, whenever a multifunction $F: X \rightarrow$ bcc $Y$ is $R$-differentiable at a point $x^{0} \in \operatorname{int}(\operatorname{dom} F)$, the limit in (15) is uniform with respect to $y^{*}$ on $B_{Y^{*}}$. Hence for each $h \in X$ the function $y^{*} \rightarrow\left\langle h, s_{F}^{\prime}\left(x^{0}, y^{*}\right)\right\rangle$ is continuous on $B_{Y^{*}}$ since it is the uniform limit of continuous functions.

Thus for $R$-differentiable multifunctions Theorem 4.6 can be strengthened as follows THEOREM 4.8 [2] If a multifunction $F: X \rightarrow \operatorname{bcc} Y$ is $R$-differentiable at a point $x^{0} \in \operatorname{int}(\operatorname{dom} F)$ then its conjugate derivative $s_{F}^{\prime}\left(x^{0}, \cdot\right): Y^{*} \rightarrow X^{*}$ is a positively homogeneous continuous mapping.

As it was observed in section 2 one can associate with a multifunction $F: X \rightarrow$ bcc $Y$ the single-valued mapping $\tilde{F}: \operatorname{dom} F \rightarrow H\left(Y^{*}\right)\left(H\left(Y^{*}\right)\right.$ is the Banach space of positive homogeneous and continuous functions from $Y^{*}$ into $\left.\mathbb{R}\right)$ defined by $\tilde{F}(x)=s_{F}(x, \cdot)$ for all $x \in \operatorname{dom} F$. Identifying the multifunction $F$ with the mapping $\tilde{F}$ we can get the following characterization of $R$-differentiability.

THEOREm 4.9 A multifunction $F: X \rightarrow$ bcc $Y$ is $R$-differentiable at a point $x^{0} \in$ $\operatorname{int}(\operatorname{dom} F)$ if and only if the single-valued mapping $\tilde{F}: \operatorname{dom} F \rightarrow H\left(Y^{*}\right)$ is classically Fréchet differentiable at the point $x^{0}$.

Moreover, the conjugate derivative $s_{F}^{\prime}\left(x^{0}, \cdot\right): Y^{*} \rightarrow X^{*}$ of a multifunction $F: X \rightarrow$ bcc $Y$ and the Fréchet derivative $\tilde{F}\left(x^{0}\right): X \rightarrow H\left(Y^{*}\right)$ are related by the equality

$$
\left\langle h, s_{F}^{\prime}\left(x^{0}, y^{*}\right)\right\rangle=\left(\tilde{F}^{\prime}\left(x^{0}\right) h\right)\left(y^{*}\right)
$$

which is satisfied for all $h \in X$ and all $y^{*} \in Y^{*}$.

Proof Straightforward. 
Since $\tilde{F}(x) \in C H\left(Y^{*}\right) \subset D C H\left(Y^{*}\right)$ for all $x \in \operatorname{dom} F$ then one can also consider $\tilde{F}$ as a mapping from dom $F$ into the normed vector space of difference-sublinear functions $D C H\left(Y^{*}\right)$. Using this fact Tyurin [3] introduced the following notion of differentiability of multifunctions.

Definition 4.10 [3] A multifunction $F: X \rightarrow$ bcc $Y$ is $\pi$-differentiable at a point $x^{0} \in$ $\operatorname{int}(\operatorname{dom} F)$ if the single-valued mapping $\tilde{F}: \operatorname{dom} F \rightarrow D C H\left(Y^{*}\right)$ is classically Fréchet differentiable at the point $x^{0}$.

$\pi$-differentiability of multifunctions was also studied by Banks and Jacobs [4] and Pecherskaya $[26,27]$. Notice that the term of $\pi$-differentiability was first introduced by Banks and Jacobs [4].

Theorem 4.11 A multifunction $F: X \rightarrow$ bcc $Y$ is $\pi$-differentiable at a point $x^{0} \in$ $\operatorname{int}(\operatorname{dom} F)$ if and only if it is $R$-differentiable at $x^{0}$ and its conjugate derivative $s_{F}^{\prime}\left(x^{0}, \cdot\right): Y^{*} \rightarrow X^{*}$ is a difference-sublinear mapping.

Proof It easily follows from Theorem 4.9 and the inclusion $D C H\left(Y^{*}\right) \subset H\left(Y^{*}\right)$.

The next theorem gives a characterization of Fréchet differentiability of multifunctions via differentiability properties of support functions.

Theorem 4.12 A multifunction $F: X \rightarrow$ bcc $Y$ is Fréchet differentiable at a point $x^{0} \in \operatorname{int}(\operatorname{dom} F)$ if and only if it is $R$-differentiable at $x^{0}$ and the function

$$
y^{*} \rightarrow s_{F}\left(x^{0}, y^{*}\right)+\left\langle h, s_{F}^{\prime}\left(x^{0}, y^{*}\right)\right\rangle
$$

is sublinear for every $h$ in some neighborhood of zero.

The Fréchet differential approximation $D F\left(x^{0} \mid \cdot\right): X \rightarrow$ bcc $Y$ of $F$ at $x^{0}$ and the conjugate derivative $s_{F}^{\prime}\left(x^{0}, \cdot\right): Y^{*} \rightarrow X^{*}$ are related to each other with the equality

$$
(D F)^{*}\left(x^{0}, y^{*}\right)=s_{F}^{\prime}\left(x^{0}, y^{*}\right) \text { for all } y^{*} \in Y^{*} .
$$

Here $(D F)^{*}\left(x^{0}, \cdot\right): Y^{*} \rightarrow X^{*}$ stands for the conjugate mapping to the affine multifunction $D F\left(x^{0}, \cdot\right): X \rightarrow$ bcc $Y$.

Proof Necessity Let $F: X \rightarrow \operatorname{bcc} Y$ be Fréchet differentiable at $x^{0} \in \operatorname{int}(\operatorname{dom} F)$ and $D F\left(x^{0} \mid \cdot\right): X \rightarrow$ bec $Y$ the Fréchet differential approximation of $F$ at $x^{0}$. Using the equality

$$
d_{H}\left(F\left(x^{0}+h\right), D F\left(x^{0} \mid h\right)\right)=\max _{\left\|y^{*}\right\| \leq 1}\left|s_{F}\left(x^{0}+h, y^{*}\right)-s_{D F}\left(x^{0} ; h, y^{*}\right)\right|
$$

and the representation of the support function $s_{D F}\left(x^{0} ; \cdot, \cdot\right)$ of $D F\left(x^{0} \mid \cdot\right): X \rightarrow$ bcc $Y$ in the form

$$
s_{D F}\left(x^{0} ; h, y^{*}\right)=s_{D F}\left(x^{0} ; 0, y^{*}\right)+\left\langle h,(D F)^{*}\left(x^{0}, y^{*}\right)\right\rangle\left(h \in \operatorname{dom} D F\left(x^{0} \mid \cdot\right), y^{*} \in Y^{*}\right)
$$

we get that the equality (10) is equivalent to

$$
\lim _{h \rightarrow 0}\left(\max _{\left\|y^{*}\right\| \leq 1} \frac{\left|s_{F}\left(x^{0}+h, y^{*}\right)-s_{F}\left(x^{0}, y^{*}\right)-\left\langle h,(D F)^{*}\left(x^{0}, y^{*}\right)\right\rangle\right|}{\|h\|}\right)=0 .
$$


It shows that the multifunction $F$ is $R$-differentiable at $x^{0}$ with $(D F)^{*}\left(x^{0} \mid \cdot\right): Y^{*} \rightarrow X^{*}$ being the conjugate derivative of $F$ at $x^{0}$. Besides we have that

$$
\begin{aligned}
s_{F}\left(x^{0}, y^{*}\right)+\left\langle h, s_{F}^{\prime}\left(x^{0}, y^{*}\right)\right\rangle & =s_{D F}\left(x^{0} ; 0, y^{*}\right)+\left\langle h,(D F)^{*}\left(x^{0}, y^{*}\right)\right\rangle \\
& =s_{D F}\left(x^{0} ; h, y^{*}\right) \text { for all } y^{*} \in X \text { and } h \in X .
\end{aligned}
$$

Hence, the function $y^{*} \rightarrow s_{F}\left(x^{0}, y^{*}\right)+\left\langle h, s_{F}^{\prime}\left(x^{0}, y^{*}\right)\right\rangle$ is, in fact, the support function of the affine multifunction $D F\left(x^{0} \mid \cdot\right): X \rightarrow \operatorname{bcc} Y$ with $0 \in \operatorname{int}\left(\operatorname{dom} D F\left(x^{0} \mid \cdot\right)\right)$. Consequently, $y^{*} \rightarrow s_{F}\left(x^{0}, y^{*}\right)+\left\langle h, s_{F}^{\prime}\left(x^{0}, y^{*}\right)\right\rangle$ is a sublinear function for all $h$ in some neighborhood of zero.

Sufficiency If a multifunction $F: X \rightarrow$ bcc $Y$ is $R$-differentiable at $x^{0} \in \operatorname{int}(\operatorname{dom} F)$ and the function defined by (17) is sublinear for each $h$ in some neighborhood of zero then the function $\left(h, y^{*}\right) \rightarrow s_{F}\left(x^{0}, y^{*}\right)+\left\langle h, s_{F}^{\prime}\left(x^{0}, y^{*}\right)\right\rangle$ is the affine extension of the support function (see Remark 3.15) of some inextensible affine multifunction $\mathcal{A}\left(x^{0} ; \cdot\right): X \rightarrow \operatorname{bcc} Y$ which satisfies Definition 4.1 and the equality (18). It completes the proof of the theorem.

Remark 4.13 From the assumption that the function defined by (17) is sublinear for each $h$ in some neighborhood of zero it follows that the conjugate derivative $s_{F}^{\prime}\left(x^{0}, \cdot\right): Y^{*} \rightarrow X^{*}$ is difference-sublinear mapping. It shows that Theorem 4.12 can be improved as follows: a multifunction $F: X \rightarrow$ bcc $Y$ is Fréchet differentiable at $x^{0} \in \operatorname{int}(\operatorname{dom} F)$ if and only if it is $\pi$-differentiable at $x^{0}$ and the function defined by (17) is sublinear for each $h$ in some neighborhood of zero.

Remark 4.14 Theorem 4.12 can be considered as an extension of Theorem 4.2 of [20] to multifunctions defined on arbitrary finite-dimensional vector spaces.

\section{References}

[1] Blagodatskikh, V.I., 1984, Maximum principle for differential inclusions. Trudy Matematicheskogo Instituta AN SSSR, 166, 23-43 (in Russian).

[2] Rubinov, A.M., 1985, The conjugate derivative of a multivalued mapping and differentiability of the maximum function under connected constraints. Sibirskii Matematicheskii Zhurnal, 26(3), 147-155 (in Russian).

[3] Tyurin, Yu. N., 1965, A mathematical formulation of a simplified model of industrial planning. Ekonomika i Matematicheskie Metody, 1(3), 391-409 (in Russian).

[4] Banks, H.T. and Jacobs, M.Q., 1970. On differential calculus of multifunctions. Journal of Mathematical Analysis and Applications, 29(3), 246-272.

[5] Aubin, J.-P. and Frankowska, H., 1990, Set-Valued Analysis, (Boston: Birkhauser).

[6] Kruger, A.Y., 1996, Strict $\varepsilon$-subdifferentials and differentiation of set-valued mappings. Doklady Akademii Nauk Belarus, 40(6), 38-43 (in Russian).

[7] Mordukhovich, B.S., 1988, Approximation Methods in Problems of Optimization and Control (Moscow: Nauka) (in Russian).

[8] Mordukhovich, B.S., 1994, Generalized differential calculus for nonsmooth and set-valued mappings. Journal of Mathematical Analysis and Applications, 183, 250-288.

[9] Mordukhovich, B.S., 1996, Nonconvex differential calculus for infinite-dimensional multifunctions. Set-Valued Analysis, 4, 205-236.

[10] Mordukhovich, B.S., 1997, Coderivatives of set-valued mappings: calculus and applications. Nonlinear Analysis, 30, 3059-3070.

[11] Mordukhovich, B.S. and Wang, B., 2002, Differentiability and regularity of Lipschitzian mappings. Proceedings of the American Mathematical Society, 131, 389-399. 
[12] Penot, J.-P., 1984, Differentiability of relations and differential stability of perturbed optimization problems. SIAM Journal of Control and Optimization, 22, 529-551.

[13] Polovinkin, E.S., 1986, The Theory of Multivalued Mappings (Moscow: Moscow Physicotechnical Institute) (in Russian).

[14] Polovinkin, E.S. and Smirnov, G.V., 1986, Differentiation of multivalued mappings and properties of solutions of differential inclusions. Soviet Mathematics Doklady, 33, 662-666.

[15] Pham Huu Sach, 1988, Differentiability of set-valued maps in Banach space. Mathematischen Nachrichten, 139, 215-235.

[16] Rockafellar, R.T., 1989, Proto-differentiability of set-valued mappings and its applications to optimization. In: H. Attouch, J.-P. Aubin, F. Clarke and I. Ekeland (Eds), Analyse Non Lineaire (Paris: Gauthier-Villars), pp. 449-482.

[17] Artstein, Z., 1995, On the calculus of set-valued maps and set-valued evolution equations. Set-Valued Analysis, 3, 213-261.

[18] de Blasi, F.S., 1976, On the differentiability of multifunctions. Pacific Journal of Mathematics, 66, 67-81.

[19] Demyanov, V.F., Lemarechal, C. and Zowe, J., 1986, Approximation of set-valued mapping I: a proposal. Journal of Applied Mathematics and Optimiztion, 14, 203-214.

[20] Lemarechal, C. and Zowe, J., 1991, The eclipsing concept to approximate a multi-valued mapping. Optimization, 22, 3-37.

[21] Martelli, M. and Vignolli, A., 1974, On differentiability of multivalued maps. Bollettino della Unione Mathematica Italiana, Serie IV, 10(3), 701-712.

[22] Silin, D.B., 1997, On set-valued differentiation and integration. Set-Valued Analysis, 5, 107-146.

[23] Le Van Hot, 1981, On the differentiability of multivalued mappings, I. Commentationes Mathematicae Universitatis Carolinae, 22(2), 267-280.

[24] Le Van Hot, 1981, On the differentiability of multivalued mappings, II. Commentationes Mathematicae Universitatis Carolinae, 22(2), 337-350.

[25] Nurminski, E.A., 1978, On differentiability of multivalued mappings. Kibernetika, 5, 46-48 (in Russian).

[26] Pecherskaya, N.A., 1981, On the differentiability of set-valued mappings. Vestnik Leningradskogo Universiteta, 7(2), 115-117 (in Russian).

[27] Pecherskaya, N.A., 1986, Quasidifferentiable mappings and the differentiability of maximum functions. Mathematical Programming Study, 29, 145-159.

[28] Blagodatskikh, V.I. and Filippov, A.F., 1986, Differential inclusions and optimal control. Proceedings of the Steklov Institute of Mathematics, 168, 199-259.

[29] Kutateladze, S.S. and Rubinov, A.M., 1976, Minkovski Duality and its Applications (Novosibirsk: Nauka) (in Russian).

[30] Rockafellar, R.T., 1970, Convex Analysis (Princeton, New Jersey: Princeton University Press).

[31] Demyanov, V.F. and Rubinov, A.M., 1986, Quasidifferential Calculus, Optimization Software (New York: Optimization Software Inc., Publication Division), p. 301.

[32] Demyanov, V.F. and Rubinov, A.M., 1990, Foundations of Nonsmooth Analysis. Quasidifferential Calculus (Moscow: Nauka) (in Russian).

[33] Demyanov, V.F. and Rubinov, A.M., 1995, Constructive Nonsmooth Analysis (Frankful am Main: Peter Lang).

[34] Gorokhovik, V.V., 1990, Convex and Nonsmooth Vector Optimization Problems (Minsk: Nawuka i Tekhnika) (in Russian).

[35] Pshenichnyi, B.N., 1974, Convex multivalued mappings and their conjugates. In: J. Loś and M.W. Loś (Eds), Mathematical Models in Economics (Warszawa: PWN-Polish Scientific Publishers), pp. 333-349.

[36] Pshenichnyi, B.N., 1980, Convex Analysis and Extremum Problems (Moscow: Nauka) (in Russian).

[37] Gautier, S., 1990, Affine and eclipsing multifunctions. Numerical Functional Analalysis and Applications, 11, 679-699.

[38] Gorokhovik, V.V. and Zabreiko, P.P. 1998, Differentiability of multi-valued mappings in sense of Fréchet. Trudy Instituta Matematiki NAN Belarusi, 1, 34-49 (in Russian).

[39] Danford, N. and Schwartz, J., 1958, Linear Operators. Part I: General Theory (New York-London: Interscience Publishers).

[40] Le Van Hot, 1985, On the open mapping principle and convex multivalued mappings. Acta Universitatis Carolinae-Mathematica et Physica, 26(1), 53-59.

[41] Makarov, V.L. and Rubinov, A.M., 1979, Mathematical Theory of Economic Dynamics and Equilibrium (New York: Springer-Verlag).

[42] Borwein, J.M., 1995, Adjoint process duality. Mathematics of Operation Research, 8(3), 403-434.

[43] Ioffe, A.D., 1981, Nonsmooth analysis: differential calculus of nondifferentiable mappings. Transactions of the American Mathematical Society, 266, 1-56. 